\title{
Role of the VirA histidine autokinase of Agrobacterium tumefaciens in the initial steps of pathogenesis
}

\author{
Yi-Han Lin ${ }^{1}$, B. Daniel Pierce ${ }^{1}$, Fang Fang ${ }^{1}$, Arlene Wise ${ }^{2}$, Andrew N. Binns ${ }^{2}$ and David G. Lynn ${ }^{1 *}$ \\ Lynn Lab, Department of Chemistry and Biology, Emory University, Atlanta, GA, USA \\ 2 Binns Lab, Department of Biology, Plant Sciences Institute, University of Pennsylvania, Philadelphia, PA, USA
}

\section{Edited by:}

Stanton B. Gelvin, Purdue

University, USA

Reviewed by:

Franz Narberhaus, Ruhr University

Bochum, Germany

Stephen Winans, Cornell University, USA

*Correspondence:

David G. Lynn, Department of Chemistry and Biology, Emory

University, 1515 Dickey Drive,

Atlanta, GA 30322, USA

e-mail:dlynn2@emory.edu
Histidine kinases serve as critical environmental sensing modules, and despite their designation as simple two-component modules, their functional roles are remarkably diverse. In Agrobacterium tumefaciens pathogenesis, VirA serves with VirG as the initiating sensor/transcriptional activator for inter-kingdom gene transfer and transformation of higher plants. Through responses to three separate signal inputs, low $\mathrm{pH}$, sugars, and phenols, A. tumefaciens commits to pathogenesis in virtually all flowering plants. However, how these three signals are integrated to regulate the response and why these signals might be diagnostic for susceptible cells across such a broad host-range remains poorly understood. Using a homology model of the VirA linker region, we provide evidence for coordinated long-range transmission of inputs perceived both outside and inside the cell through the creation of targeted VirA truncations. Further, our evidence is consistent with signal inputs weakening associations between VirA domains to position the active site histidine for phosphate transfer. This mechanism requires long-range regulation of inter-domain stability and the transmission of input signals through a common integrating domain for VirA signal transduction.

Keywords: VirA, GAF domain, signal transduction, pathogenesis, two-component system, Agrobacterium

\section{INTRODUCTION}

Dynamic fluctuations in conformation can be essential for protein function, and large-scale adjustments are often necessary for complex cellular events ranging from allosteric enzymatic activity, regulation of overlapping signal transduction pathways, and the many intra- or inter-subunit protein-protein, proteinDNA, and protein-RNA interactions associated with information flow (Chillemi et al., 2003; Laskowski et al., 2009; Farago et al., 2010). Such protein dynamics are not typically highlighted in static structural models, but can be of critical importance to our understanding of function. The complex roles of the membranebound histidine kinases, which function as receptors and signal transducers to modify gene expression or protein function in response to environmental change in many prokaryotes, are critical for committing Agrobacterium tumefaciens to pathogenesis (Stock et al., 2000; Mitrophanov and Groisman, 2008; Cheung and Hendrickson, 2010).

The VirA histidine kinase and its response regulator VirG form a two-component stimulus-response coupling pair (Gelvin, 2000; Lin et al., 2008). This pair is the necessary first step in the regulation of transcription of the virulence (vir) genes on the tumor inducing $(\mathrm{Ti})$ plasmid that ultimately mediate the transfer and integration of DNA into the host cell (Gelvin, 2006; Tzfira and Citovsky, 2006). The multi-domain VirA kinase (Figure 1A) exists as a transmembrane dimer (Pan et al., 1993; Brencic et al., 2004; Wise et al., 2005) and responds to a broad range of phenols (Melchers et al., 1989; Duban et al., 1993) and monosaccharides in low $\mathrm{pH}$ environments (Ankenbauer and Nester, 1990; Brencic et al., 2004; Wise et al., 2005; Hu et al., 2013). Maximal expression of the vir genes requires a $\mathrm{pH}$ sensitive monosaccharide binding to a periplasmic protein ChvE (Ankenbauer and Nester, 1990; Cangelosi et al., 1990). Both ChvE/sugar and phenols associate with VirA to regulate VirG phosphorylation (Chang and Winans, 1992). The terminal receiver domain of VirA homologous to VirG and has been shown to have both negative and positive effects on the phosphorylation cascade (Chang et al., 1996; Wise et al., 2010). Therefore, coordinated actions across the entire VirA dimer appears to be necessary for signal perception and transmission. The central position of the "linker" domain, which joins the trans-membrane helices to the kinase domain, suggests that both periplasmic and cytoplasmic inputs might be integrated here for transmission to the catalytic histidine 474 , which is phosphorylated and subsequently used to phosphorylate VirG (Chang and Winans, 1992).

We have used homology models of the VirA linker to gain mechanistic insight for long-range conformational regulation of VirA activity (Wang et al., 2002; Gao and Lynn, 2007). Using mutational and chimeric protein constructs to test prediction, we now document specific interactions within and between VirA domains critical for signal transmission. These long-range structural interactions reveal additional insights into the integrator functions of the linker domain. While it is not yet clear how general these insights may be or why these specific signal inputs have been selected for broad host range evolution, it is certainly clear that sophisticated cooperative motions throughout the entire sensor kinase are exploited for the successful pathogenesis by Agrobacterium tumefaciens. 
A

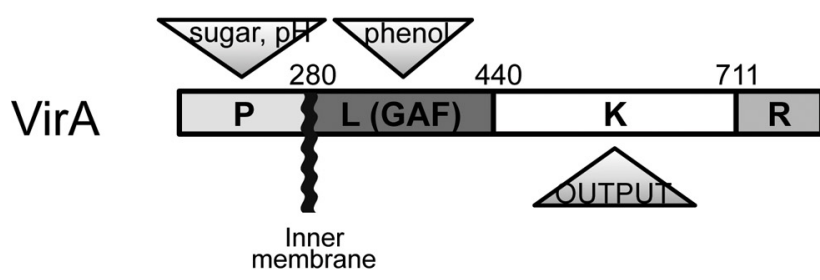

B

VirA $\quad 292-441$

$3 \mathrm{mmhA} \quad 15-161$

VirA $292-441$

3mmh $15-161$

VirA 292-441

$3 \operatorname{mmhA} \quad 15-161$

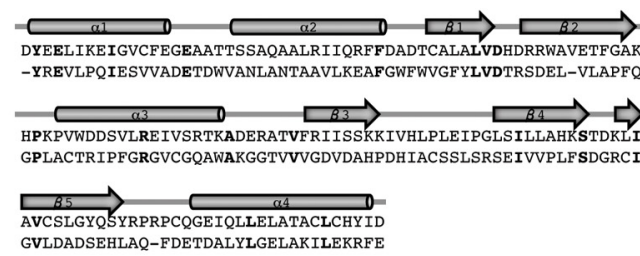

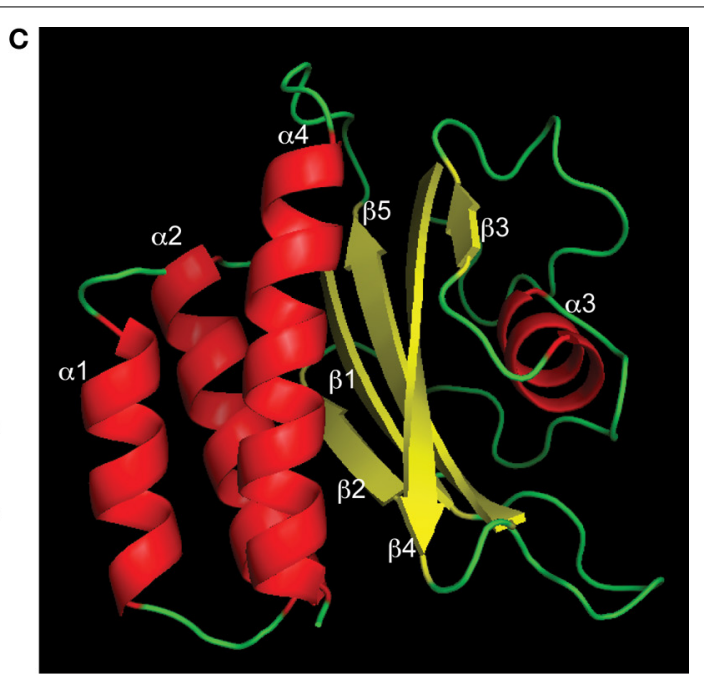

FIGURE 1 | Domain architecture of the histidine autokinase VirA. (A) Domain organization and signal inputs of VirA. Besides the conserved kinase (K), three regulatory domains exist to coordinate the signal output. The periplasmic domain $(P)$ perceives sugar and $\mathrm{H}^{+}$, the linker domain senses the phenol, and a receiver domain $(R)$ locates at the $\mathrm{C}$-terminus for additional regulation. (B)
Homology between VirA (292-441) and the Neisseria meningitidis fRMsr protein, using Phyre2 (Kelley and Sternberg, 2009). Bold lettering indicates identical residues. (C) Predicted structure of the VirA linker region. The GAF-domain containing protein from $N$. meningitidis (PDB ID: $3 \mathrm{MMH}$ ) provided a template for a predicted protein structure of the VirA linker (292-441).

\section{MATERIALS AND METHODS LINKER STRUCTURE MODELING}

The VirA (292-441) sequence was used to perform a secondary structure homology search using Phyre2 (Kelley and Sternberg, 2009). The GAF domain was common to all but a few of the top 20 hits, and several of these protein structures were known (1VHM, 1F5M) (Gao and Lynn, 2007) (Figure S1A). The top hit was the fRMsr protein from Neisseria meningitidis, $12 \%$ identity with $93.4 \%$ confidence. fRMsr and other hits (e.g., 3P01 and 1F5M) were used as templates for VirA (292-441) (Figure S1B). Comparisons of the resulting GAF domains, including the previous threading of this VirA domain using Swiss Model Workspace (Gao and Lynn, 2007), provided structures that differed only slightly in the relative orientations of the secondary elements (Figure S1C).

\section{BACTERIAL STRAINS, PLASMIDS, AND REAGENTS}

The bacterial strains and plasmids used in this study are listed in Table 1. E. Coli strain XL1-Blue (Strategene) was used for routine plasmid construction. Acetosyringone (AS) used for vir gene induction was purchased from Sigma-Aldrich Corp. Isopropyl $\beta$ D-1-thiogalactopyranoside (IPTG) used to induce protein expression and 5-bromo-4-chloro-3-indolyl-beta-D-galactopyranoside (X-gal) used in library screening were purchased from Research Products International Corp. All cloning reagents were purchased from either New England Biolab or Promega.

\section{PLASMID CONSTRUCTIONS}

While the scheme for the design of constructs is shown in Figure 2B, plasmid construction procedures are described in Supplementary Materials. The plasmids are listed in Table 1, and the primers are listed in Table S2.

\section{LIBRARY CONSTRUCTION AND SCREENING}

The constitutively active mutants in $\alpha 4$ were identified by randomly mutating aa426-437 in $\operatorname{LKR}(285-829)$ via two-step PCR using the primers with an NNN codon replacing each residue, and the results being amplified using primers LKR285 (5'CGGGATCCGATTGGTTAGCGCGGCGT-3') and LKRA1 ( $5^{\prime}$ GCGGTACCGCAACTCTACGTCTTGAT-3'). The library was digested with BamHI and Acc65I and ligated into the BamHI and Acc65I digested pJZ6. These constructs were directly transformed into A. tumefaciens strain A136 containing pRG109 by eletroporation. To select for the constitutively "on" variants, the transformants of the mutated aa426-437 library were screened on non-inducing media plates containing X-gal. The blue colonies were extracted, sequenced, and the phenotype confirmed by site-directed mutagenesis.

\section{及-GALACTOSIDASE ASSAYS FOR Vir GENE INDUCTION}

The GCN4 leucine zipper variants, $\mathrm{LZ}(\mathrm{n})-426^{\mathrm{K}(\mathrm{G} 665 \mathrm{D})}$, were transformed into A. tumefaciens strain A348-3 containing pRG150, which has $l a c I^{\mathrm{q}}$ to allow chimera expression only during IPTG induction. The A. tumefaciens strains were grown in $\mathrm{LB}$ medium with appropriate antibiotics at $28^{\circ} \mathrm{C}$ to an $\mathrm{OD}_{600}$ of $0.4-0.6$. The cells were pelleted by centrifugation at $4^{\circ} \mathrm{C}, 7000 \times$ $\mathrm{g}$, for $10 \mathrm{~min}$. The pellet was washed with PBS, and diluted to $\mathrm{OD}_{600} \sim 0.1$ into tubes containing a total of $1 \mathrm{~mL}$ induction medium (Winans et al., 1988) with $200 \mu \mathrm{M}$ IPTG, and cultured at $28^{\circ} \mathrm{C}, 225 \mathrm{rpm}$ for $15 \mathrm{~h}$. $\beta$-galactosidase activity was determined as previously described (Miller, 1972), and the reading of optical densities at 600 and $415 \mathrm{~nm}$ was performed using a EL800 microplate reader (BIO-TEK Instruments).

Except for the $\mathrm{LZ}(\mathrm{n})-426^{\mathrm{K}(\mathrm{G} 665 \mathrm{D})}$ variants, all of the virA variants and fusions were transformed into A. tumefaciens strain A136 containing pRG109, which carries $P_{\text {virB }}-l a c Z$ and $P_{N 25}-v i r G$, for 
Table 1 | Bacterial strains and plasmids used in this study.

\begin{tabular}{|c|c|c|}
\hline Strains/plasmids & Relevant characteristics & References \\
\hline XL1-Blue & recA1 endA1 gyrA96 thi-1 hsdR17 supE44 relA1 lac[ $F^{\prime}$ proAB lacla Z M15 Tn $\left.10\left(\operatorname{Tc}^{r}\right)\right]$ & Stratagene \\
\hline A136 & Strain C58 cured of pTi plasmid & Watson et al., 1975 \\
\hline A348-3 & A136 containing pTiA6NC, $\triangle$ PvirA - virA, virA deletion, $\mathrm{Km}^{r}$ & Lee et al., 1992 \\
\hline pYW33 & PN25-6xHis-LZ-virA(aa285-471) in pYW15, Ap ${ }^{r}$ & Wang et al., 2002 \\
\hline pYW39 & PN25-6xHis-virA(aa285-829)(G665D) in pYW15, Ap ${ }^{r}$ & Wang et al., 2000 \\
\hline pYW48 & 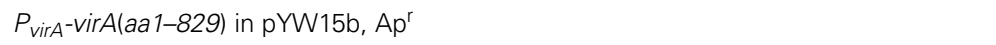 & Wang et al., 2000 \\
\hline pSW209 $\Omega$ & virB::lacZ, IncP, Spec ${ }^{r}$ & Wang et al., 2000 \\
\hline pJZ4 & $P_{\text {virB-lacZ in pMON596, IncP Spec }}{ }^{r}$ & Zhang et al., 2000 \\
\hline pRG179 & 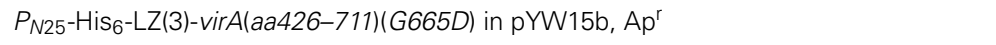 & Gao and Lynn, 2007 \\
\hline pRG180 & 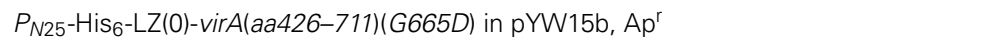 & Gao and Lynn, 2007 \\
\hline pYL28 & $P_{N 25}-H_{i s}-v i r A(a a 285-829)(C 435 F)$ in $p J Z 6, A^{r}$ & This study \\
\hline pYL64 & 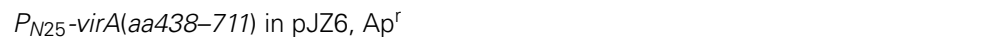 & This study \\
\hline pYL75 & $P_{N 25}-$ virA(aa285-711) in pJZ6, $A p^{r}$ & This study \\
\hline pYL81 & $P_{N 25}$-virA(aa446-711) in pJZ6, $\mathrm{Ap}^{r}$ & This study \\
\hline pYL99 & $P_{N 25}-$ virA(aa426-711) in pJZ6, $A p^{r}$ & This study \\
\hline pYL100 & $P_{N 25}$-virA(aa460-711) in pJZ6, $\mathrm{Ap}^{r}$ & This study \\
\hline pYL102 & $P_{N 25}$-virA(aa453-711) in pJZ6, $\mathrm{Ap}^{\mathrm{r}}$ & This study \\
\hline pYL103 & 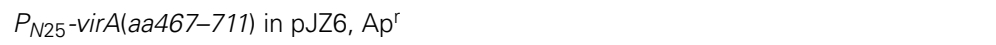 & This study \\
\hline pYL148 & $P_{N 25-v i r A(a a 426-711)(Q 427 W)}$ in pJZ6, Ap ${ }^{r}$ & This study \\
\hline pYL149 & $P_{N 25}$-virA(aa426-711)(E430K) in pJZ6, Ap ${ }^{r}$ & This study \\
\hline pYL150 & 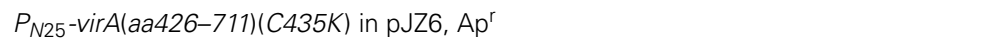 & This study \\
\hline pYL200 & $P_{N 25}$-LZ(4)-virA(aa450-829) in pJZ6, $\mathrm{Ap}^{r}$ & This study \\
\hline pYL201 & $P_{N 25}$-LZ(3)-virA(aa450-829) in pJZ6, Ap ${ }^{r}$ & This study \\
\hline pYL202 & $P_{N 25}$-LZ(0)-virA(aa450-829) in pJZ6, $\mathrm{Ap}^{r}$ & This study \\
\hline pYL203 & 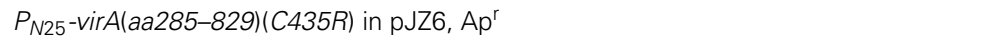 & This study \\
\hline pYL205 & 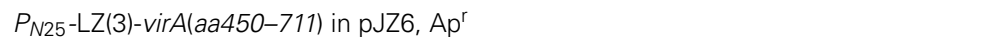 & This study \\
\hline pYL206 & $P_{N 25}$-LZ(0)-virA(aa450-711) in pJZ6, $A p^{r}$ & This study \\
\hline pYL207 & $P_{N 25}$-LZ(4)-virA(aa450-711) in pJZ6, Ap ${ }^{r}$ & This study \\
\hline pYL212 & 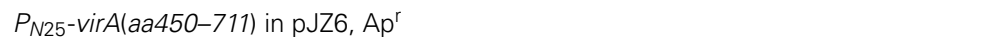 & This study \\
\hline pYL213 & $P_{N 25}$-virA(aa450-829) in pJZ6, $A^{r}$ & This study \\
\hline pYL214 & $P_{N 25}$-LZ(-2)-virA(aa450-829) in pJZ6, $\mathrm{Ap}^{r}$ & This study \\
\hline pYL215 & $P_{N 25}$-LZ(-1)-virA(aa450-829) in pJZ6, $\mathrm{Ap}^{r}$ & This study \\
\hline pYL267 & 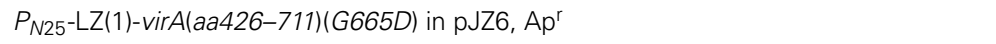 & This study \\
\hline pYL268 & 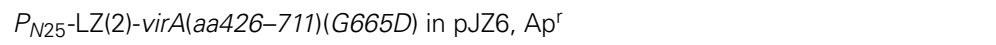 & This study \\
\hline pYL269 & $P_{N 25}$-LZ(-1)-virA(aa426-711)(G665D) in pJZ6, $A p^{r}$ & This study \\
\hline pYL270 & $P_{N 25}$-LZ(-2)-virA(aa426-711)(G665D) in pJZ6, $\mathrm{Ap}^{r}$ & This study \\
\hline pYL283 & 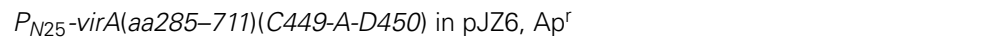 & This study \\
\hline
\end{tabular}


Table 1 | Continued

\begin{tabular}{|c|c|c|}
\hline Strains/plasmids & Relevant characteristics References & \\
\hline pYL295 & 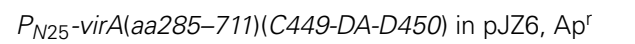 & This study \\
\hline pYL296 & $P_{N 25}$-virA(aa285-711)(C449-DALK-D450) in pJZ6, Ap ${ }^{r}$ & This study \\
\hline pYL306 & $P_{N 25}$-virA(aa285-711)(C449-DAL-D450) in $\mathrm{pJZ6}, \mathrm{Ap}^{r}$ & This study \\
\hline pYL307 & $P_{N 25}$-virA(aa285-829)(K298E) in pJZ6, Ap $^{r}$ & This study \\
\hline pYL308 & 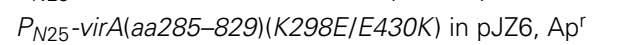 & This study \\
\hline
\end{tabular}

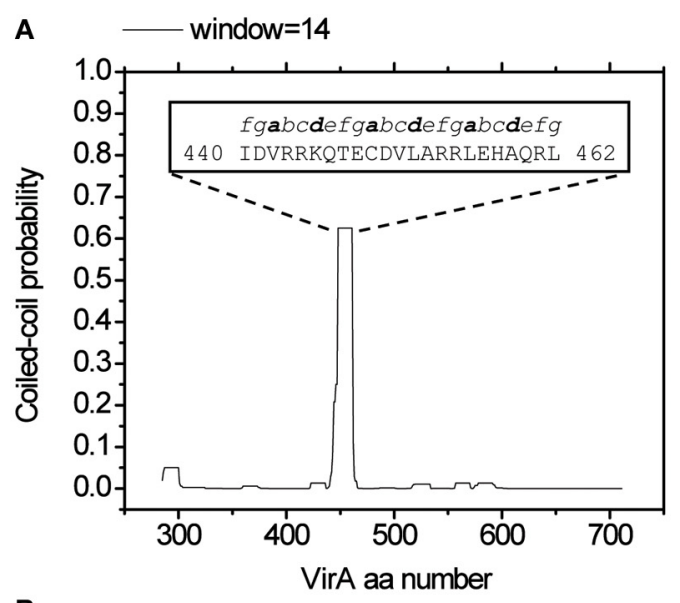

B

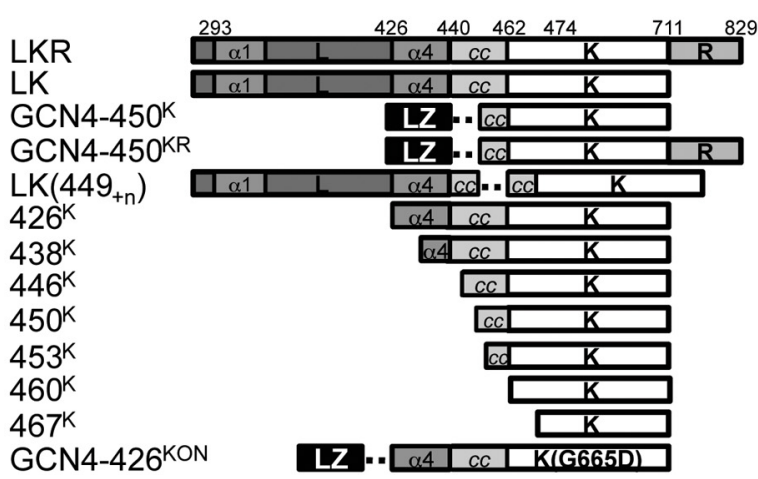

C

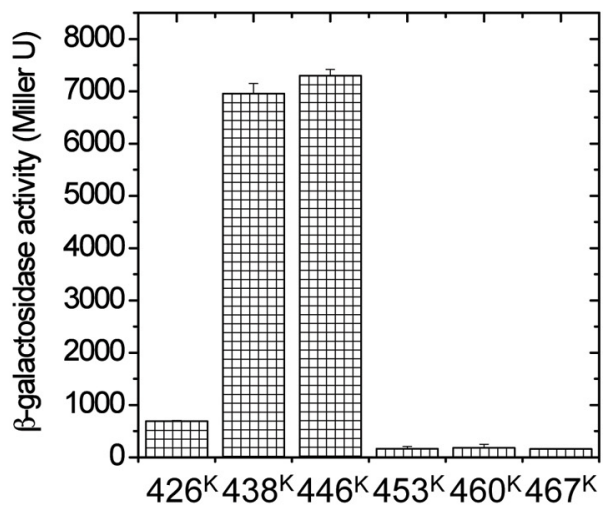

Expression:
FIGURE 2 | Design of the VirA variants. (A) COILS was used to predict coiled-coil forming propensity of VirA-LK(285-711). Aa440-462 was predicted to have high coiled-coil forming probability, and the predicted heptad repeats is shown inside the figure. Heptad positions a and $d$ are shown in bold for orientation. (B) The design of VirA truncations, GCN4 fusions, kinase

truncations, and direct amino acid insertions. The predicted $\alpha 1$ and $\alpha 4$ of the linker domain and the coiled-coil in $\mathrm{K}$ are shown with the dashed line indicating the inserted adapter. (C) $\beta$-galactosidase activity of different kinase truncations. A. tumefaciens strain A136 carrying pRG109 and the kinase truncations from $426^{\mathrm{K}}$ to $467^{\mathrm{K}}$ were assayed for vir gene expression in the absence of inducers. In vivo protein expression of each truncation was analyzed by Western blot and shown below. vir gene expression. The cells were grown and pelleted by the same procedure described above, and diluted to $\mathrm{OD}_{600} \sim 0.1$ into tubes containing a total of $1 \mathrm{~mL}$ induction media with or without $300 \mu \mathrm{M}$ AS, as indicated, and cultured at $28^{\circ} \mathrm{C}, 225 \mathrm{rpm}$ for $15 \mathrm{~h}$. The $\beta$-galactosidase activity was determined by the same method as described, from the reading of the optical densities at 600 and $415 \mathrm{~nm}$.

\section{IMMUNOBLOT ANALYSIS}

A. tumefaciens strains were grown in $50 \mathrm{~mL} \mathrm{LB}$ medium with appropriate antibiotics at $28^{\circ} \mathrm{C}$ overnight. The cells were harvested by centrifugation at $4^{\circ} \mathrm{C}, 7000 \times \mathrm{g}$, for $10 \mathrm{~min}$. The pelleted cells were washed with PBS and lysed on ice by sonication. The clear lysates were obtained by centrifugation at $4^{\circ} \mathrm{C}, 9000 \times \mathrm{g}$, for $10 \mathrm{~min}$, and analyzed by $10 \%$ SDS-PAGE followed by electro-blotting onto nitrocellulose membrane. The membrane was blocked with 3\% BSA in TBS, and probed with anti-VirA polyclonal antibody (see SI methods) at 1:200 dilutions. Visualization was achieved using the goat anti-rabbit antibody conjugated with alkaline phosphatase (Amersham) at 1:1000 dilutions, followed by the 1-step NBT/BCIP development (Pierce).

\section{RESULTS}

\section{STRUCTURAL MODEL FOR THE LINKER DOMAIN OF VirA}

The linker domain, designated (L) as it connects TM2 (ending at aa279) with the kinase (K) domain of VirA 
(Chang and Winans, 1992), was originally defined through mutagenesis and sequence analyses as responsible for phenol signal regulation of kinase activity (Figure 1A). Conservatively selecting residues 292-441 for a Phyre 2 secondary structure search revealed $85 \%$ of the top 20 hits as GAF domains, so named because of their presence in $\mathrm{c}$ GMP-regulated cyclic nucleotide phosphodiesterases, $\underline{A}$ denylyl cyclases, and the bacterial transcription factor FhlA (Kelley and Sternberg, 2009). Several of these proteins have structural models, and of these, the GAF-domain containing protein fRMsr from Neisseria meningitis is the most similar (Gruez et al., 2010). While previous GAF domain-containing proteins are homologous to the VirA linker (Gao and Lynn, 2007), the fRMsr protein (PDB ID: $3 \mathrm{MMH}$ ) provides a stronger template with $93.4 \%$ confidence at $98 \%$ coverage, defining the relative positioning of the $\alpha$-helices and $\beta$-sheets (Figure 1B). Using other protein structures as templates gave similar structures with only slight changes in the orientation of the conserved secondary elements (Figure S1).

The resulting threading model of the VirA linker region (Figure 1C) contains a central $\beta$-sheet, arranged in a 2-1-5-43 strand order (Figure 1C), connected to a helix bundle region composed of $\alpha 1, \alpha 2$, and $\alpha 4$ that connects the linker region to the histidine kinase (see Figure 1A). A four-helix bundle architecture, similar to the proposed bundle in VirA, has been characterized in HAMP domains (derived from $\underline{H}$ istidine kinases, $\underline{A}$ denyl cyclases, $\underline{M}$ ethyl-accepting proteins, and $\underline{P}$ hosphatases) (Aravind and Ponting, 1999). These domains regulate signal transmission in histidine kinases (HK) (Falke and Hazelbauer, 2001; Hulko et al., 2006; Airola et al., 2010) and are thought to constitute a dimerization interface (Gao and Lynn, 2007). The $\alpha 1, \alpha 2$, and $\alpha 4$ helix region of VirA is proposed to serve as the interface in the VirA dimer based on homologies with these domains.

Initial physical analyses of this model involved over-expressing and purifying the N-terminal $\mathrm{His}_{6}$-tagged VirA (285-471) domain (Figure S2A). The relative abundances of secondary structure determined by circular dichroism supported the threading model ( $\sim 34 \% \alpha$-helix, $\sim 20 \% \beta$-sheet), but conditions were not found to sufficiently stabilize this truncated domain for further evaluation (Figure S2B). Additional sequence analysis of the full LK domains of VirA (285-711) with COILS (Lupas et al., 1991) identified strong coiled-coil propensity connecting the GAF fold to the N-terminus of the DHp domain, a region in Thermotoga maritima HK0853 and Saccharomyces cerevisiae Sln 1 critical for signal transmission (Tao et al., 2002; Marina et al., 2005). Employing several scanning windows of the heptad repeats, COILS identified aa440-462 (Figure 2A) as having an amphipathic heptad repeat signature. Increasing the size of the scanning window lowered the probability of this region as a coiled-coil, presumably because the sequences surrounding this region do not contribute to the coiled-coil.

To directly evaluate the role of the predicted coiled-coil, we constructed a series of N-terminal truncations of the kinase domain (Figure 2B), starting from amino acid $426\left(426^{\mathrm{K}}\right)$, which includes the entire $\alpha 4$ of the linker (L) domain (Gao and Lynn, 2007 ) and extending through amino acid $467\left(467^{\mathrm{K}}\right)$ for complete coiled-coil removal. Most of these truncations appeared stable, but the immunoblot suggests that constructs where the coiled-coil is removed are expressed in lower amounts. To examine how well these VirA fragments are able to induce the vir genes, we used a well-characterized $\beta$-galactosidase assay where the VirB promoter is placed in front of a plasmid localized lac $Z$ gene. As VirA receives the phenol signal, the VirB promoter is turned on and $\beta$-galactosidase is produced from the lac $Z$ gene. The $\beta$-gal activity can then be assayed using its cleavage of the substrate ONPG (Miller, 1972), thereby effectively revealing the activity of the VirA protein. In the absence of inducers, VirA fragments $438^{\mathrm{K}}$ and $446^{\mathrm{K}}$, which retain all or most of the coiled-coil region, have high activity, while partial $\left(453^{\mathrm{K}}\right)$ and complete coiled-coil deletion $\left(460^{\mathrm{K}}\right.$ and $\left.467^{\mathrm{K}}\right)$ are expressed in lower amounts and have a lower activity (Figure 2C). The reduced activity of $426^{\mathrm{K}}$ is striking and consistent with previous evidence that related HAMP-like domains can also be repressive (Gao and Lynn, 2007), suggesting that the 11 amino acids (aa426-437) in $\alpha 4$ contribute to that repression when inducers are absent.

\section{FUNCTIONALLY CONNECTING THE L AND K DOMAINS}

The helix bundle architecture at the dimerization interface of the GAF-fold in the L domain and the predicted coiled-coil connection to $\mathrm{K}$ implies a continuous helical connection being necessary for signal transmission. Previous work describing incremental fusion chimeras with the yeast GCN4 coiled-coil at aa426, just before $\alpha 4$, was interpreted as anchoring the relative position of the helices of the VirA dimer (Wang et al., 2002; Gao and Lynn, 2007). The aa440-462 coiled-coil, however, suggests that in-register fusions with GCN4 are possible, allowing us to define the relative registry of each VirA monomer through to the position of the active site histidine. Fusions were therefore engineered at aa450, removing the $\mathrm{N}$-terminal half of the predicted coiledcoil (Figures 2B, 3A), and placing the fusion just 24 residues upstream of the phosphorylated His474.

While similar results were found using the $450^{\mathrm{K}}$ construct and GCN4 fusions (Figure S3), the effect of helix positioning was more dramatic when the receiver $(\mathrm{R})$ domain is retained in the constructs (Figure 3B). In our experimental conditions, where the constitutive T5 promoter drives VirG expression, the $\mathrm{R}$ domain acts as a repressor. The protein expression appeared to be enhanced in all LZ- $450^{\mathrm{KR}}$ fusions compared to $450^{\mathrm{KR}}$. The $450^{\mathrm{KR}}$ truncation was active, but the in-register $\mathrm{LZ}(0)-450^{\mathrm{KR}}$ fusion, which is predicted to place the His474 at the same $e$ heptad position, gives a 4 -fold increase in activity that may be partially attributed to increased stabilization. A three amino acid insertion, $\mathrm{LZ}(3)-450^{\mathrm{KR}}$, creating a $-51^{\circ}$ rotation relative to $\mathrm{LZ}(0)-450^{\mathrm{K}}$ and moving His474 to the $a$ heptad position, shows five times the activity of $\mathrm{LZ}(0)-450^{\mathrm{KR}}$. A four amino acid insertion, $\mathrm{LZ}(4)$ $450^{\mathrm{KR}}$, creating $\mathrm{a}+51^{\circ}$ rotation and positioning His 474 at $b$, shows the same level of kinase activity as $\mathrm{LZ}(0)-450^{\mathrm{KR}}$. The "ON" and "OFF" states being regulated by the relative position of the active site His474 was further tested with $\mathrm{LZ}(-1)-450^{\mathrm{KR}}$ and $\mathrm{LZ}(-$ 2) $-450^{\mathrm{KR}}$ constructs, corresponding to a rotation of His 474 to the $d$ and $c$ positions on the opposite face of the coiled-coil, and these fusions also showed little activity (Figure 3B).

This model was finally tested by direct insertion of amino acids at residue 449 in the center of the predicted coiled-coil, here denoted as $\operatorname{LK}\left(449_{+n}\right)$ where $n$ is the number of amino 
acids inserted (Figure 4A). As seen in Figure 4B, a +3 amino acid insertion would extend the coiled-coil by almost a single turn and create $\mathrm{a}-51^{\circ}$ rotation, most similar to the LZ(3) fusions, and a +4 amino acid insertion would extend the coiled-coil by more than one turn and create $\mathrm{a}+51^{\circ}$ rotation. The activity observed in $\operatorname{LK}\left(449_{+1,2,3,4}\right)$ constructs follows the general pattern as the GCN4 chimeras in Figure 3, but the predicted registry is different; the $+51^{\circ}$ rotation $\mathrm{LK}\left(449_{+4}\right)$ enhanced kinase activity while the $-51^{\circ}$ rotation $\operatorname{LK}\left(449_{+3}\right)$ reduced activity (Figure $\left.4 \mathrm{C}\right)$. We have no direct evidence that the positional variability is due to difference in expression or stability, nor do we know whether the inserts "buckle" or bend the helices in some way to transmit slightly different positional information down the helix to the histidine, and these assignments will require higher structural resolution.

\section{MAPPING THE HELIX ASSOCIATION INTERFACES}

To further investigate GCN4 fusions for controlling the dimer interface of $\alpha 4-\alpha 4^{\prime}$, the domain was placed outside of the coiled coil region in $\mathrm{LK}$ to create GCN4(n) $-426^{\mathrm{K}}$ with the same amino acid inserts as in Figure 3A. Since the wild-type $426^{\mathrm{K}}$ has low basal activity (Figure 2C), possibly due to repressive dimer association, a constitutive mutation, G665D, denoted as $426^{\mathrm{KON}}$, was used as before to increase basal activity (Chang et al., 1996; Gao and Lynn, 2007). The full range of GCN4- $426^{\mathrm{K}}$ fusions, $\mathrm{LZ}(0 / 1 / 2 /-1 /-2 / 3 / 4)-426^{\mathrm{KON}}$, mapped the possible rotations, and as shown in Figure 5, the activity again follows heptad orientation positioning. The highest activity was found for $\mathrm{LZ}(1)$ $426^{\mathrm{KON}}$, and the activity gradually diminished with rotations in either direction. By this analysis, $\mathrm{LZ}(1)-426^{\mathrm{ON}}$ was assigned as the lowest energy 0 degree rotation interface, and the $\alpha 4-\alpha 4^{\prime}$

$$
\begin{aligned}
& \text { A } \\
& \text { LZ insert } \quad \text { K * } \quad \text { R } \\
& \text { LZ (4) }-450^{\mathrm{K} / \mathrm{KR}} \begin{array}{c}
\text { cdefgabcdefgabcd b } \\
- \text { RLKKLVDALKDVLARR-- }
\end{array} \\
& \text { cdefgabcd efgabc a } \\
& \mathrm{LZ}(3)-450^{\mathrm{K} / \mathrm{KR}} \quad--\mathrm{RLKKLVDAL}-\mathrm{DVLARR}--\mathrm{H}_{474}--
\end{aligned}
$$

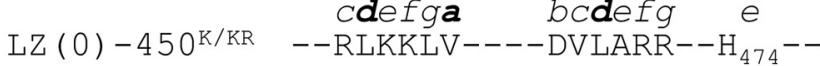

$$
\begin{aligned}
& \text { cdefg abcdef } \boldsymbol{d} \\
& \mathrm{LZ}(-1)-450^{\mathrm{K} / \mathrm{KR}}--\mathrm{RLKKL}-----\mathrm{DVLARR}--\mathrm{H}_{474}-- \\
& \text { cdef } \\
& \mathrm{LZ}(-2)-450^{\mathrm{K} / \mathrm{KR}}--\mathrm{RLKK}------ \text { DVLARR-- } \mathrm{H}_{474}-- \\
& 450^{\mathrm{K} / \mathrm{KR}} \\
& \text { bcdefg e } \\
& \text {-DVLARR-- } \mathrm{H}_{474}-\text { - }
\end{aligned}
$$

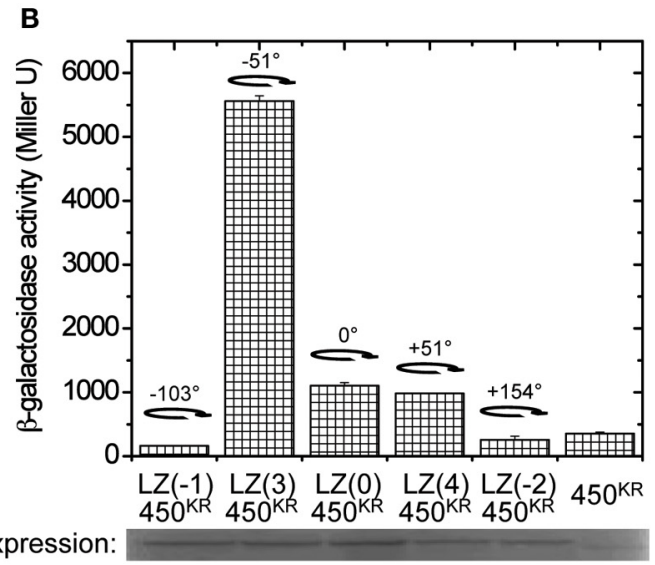

FIGURE 3 | Chimeric GCN4 fusions with $450^{\mathrm{K}}$ and $\mathbf{4 5 0} 0^{\mathrm{KR}}$. (A) Design of the GCN4-450 fusions. The heptad repeats from a to $g$ were built from the registry of GCN4 and the adapters. GCN4 enforces the hydrophobic ad interface (shown in bold), and shifts the registry of the heptads of kinase coiled-coil according to the different adapters. The predicted position of His $474(*$ in the K domain) in each fusion is shown at the end of the sequence. (B) A. tumefaciens strain A136 carrying pRG 109 and the indicated GCN4-450 KR fusions were assayed for vir gene expression without inducers. The degree of rotation created by each fusion is shown in the figure with the $0^{\circ}$ rotation defined at $L Z(0)-450$. The protein expression of the GCN4-450 KR constructs was analyzed by Western blot.

A

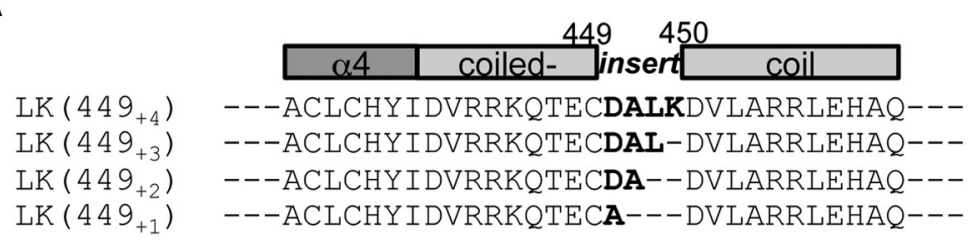

B
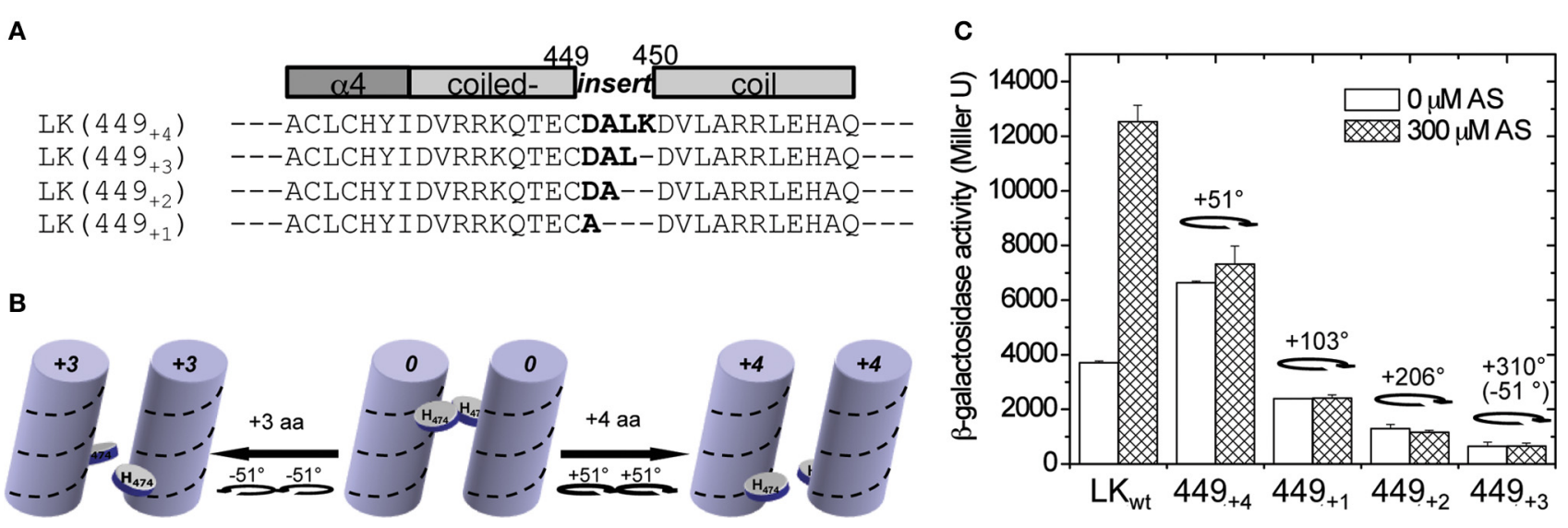

FIGURE 4 | Direct amino acid insertion within the coiled-coil. (A) The amino acids in bold were inserted between amino acids 449 and 450 in the predicted coiled-coil region. (B) An illustration of how His 474 moves along the helix coil according to the amino acid insertion at the $\mathrm{N}^{\prime}$-terminus. (C) $A$. tumefaciens strain A136 carrying pRG109 and the LK constructs with different insertions at aa449 $\left(449^{+n}\right)$ were assayed for vir gene expression with or without $300 \mu \mathrm{M}$ AS. The degree of rotation created by the insertions is shown in the graph. 
dimer interface can be designated as the "ON" conformation (Figure 8A).

The possibility of interactions across $\alpha 4-\alpha 4^{\prime}$ between subunits of the dimer suggests that inputs from sugar/ChvE association might also be transmitted through $\alpha 1$ to the dimer interface in the GAF structure. To test this possibility, we first sought "ON" interface stabilizing mutations within $\alpha 4$ that could provide signal-independent activity. Residues 426-437 of LKR (aa285829 ) were randomly mutagenized, and the variants were screened in A136/pRG109 on AB media plates with X-gal without phenolic inducer for active mutants. This approach yielded six constitutive mutants: three with substitutions at Cys435 (C435K, C435R, and C435F), two at Gln427 (Q427W and Q427F), and one at Glu430 (E430K).

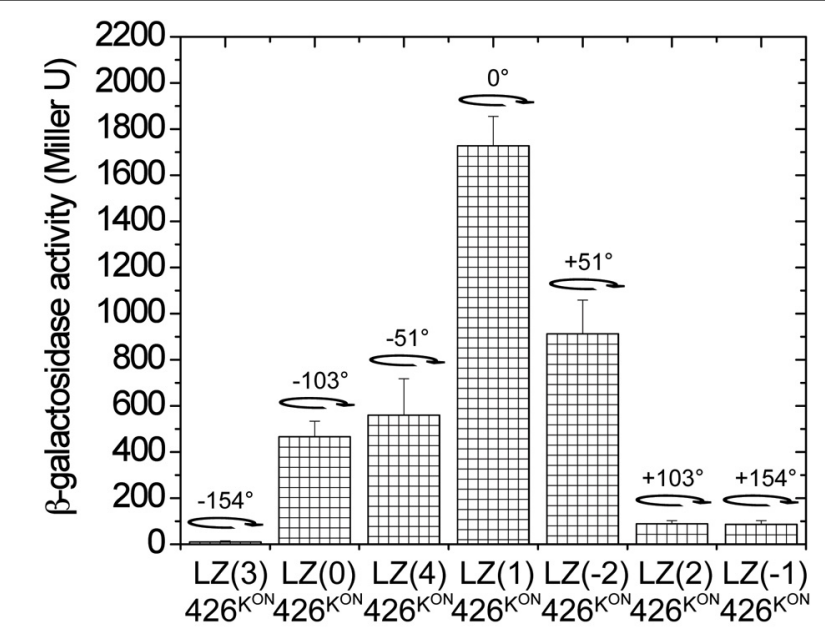

Expression:

FIGURE 5 | Signal transmission through $\alpha$ 4. $\beta$-galactosidase activity of the GCN4-426 K(G665D) fusions. A. tumefaciens strain A348-3 carrying pRG150 and the indicated GCN4-426 ${ }^{\mathrm{KON}}$ fusions were assayed for vir gene expression without inducers. $200 \mu$ M IPTG was added to induce chimera expression. The degree of rotation created by each fusion is shown in the graph with the $0^{\circ}$ rotation defined at $L Z(1)-426^{\mathrm{KON}}$
In all of these mutants, phenol induction is severely attenuated (Figure 6A), consistent with the "ON" interface being conformationally stabilized. These mutations were moved to $426^{\mathrm{K}}$ as shown in Figure 6B. The hydrophobic constitutive variants (Q427F, Q427W, C435F) and the charged variant C435K enhanced $426^{\mathrm{K}}$ activity, consistent with stabilization of the "ON" $\alpha 4-\alpha 4^{\prime}$ dimer interface, but the low basal activity of $426^{\mathrm{K}}(\mathrm{E} 430 \mathrm{~K})$ suggests that its constitutive phenotype in LKR is unlikely a result of $\alpha 4-\alpha 4^{\prime}$ stabilization. The GAF models place several charged residues distributed at the helical surface of $\alpha 1$ (Figure 7A), suggesting that the constitutive phenotype of E430K might result from $\alpha 4$ to $\alpha 1$ charge interaction. While the relative positions of these helices is weakly constrained by these modeling algorithms, among the charged residues in $\alpha 1, \mathrm{~K} 298$ is positioned close enough to form a salt-bridge with E430 in all three models with the allowance of a simple clockwise rotation. To test this possibility, a K298E mutation was constructed to complement E430K. While neither of the single $\mathrm{E} 430 \mathrm{~K}$ or $\mathrm{K} 298 \mathrm{E}$ mutations were phenol responsive, the double mutant (K298E/E430K) restored both kinase activity and phenol inducibility (Figure 7B). This compensating mutation is consistent with an $\alpha 1$ and $\alpha 4$ interface impacting signal transmission, possibly connecting sugar/ChvE binding and phenol induction to conformational transmission through this helical bundle (Gao and Lynn, 2007).

\section{DISCUSSION}

Available protein structures and comparison algorithms have dramatically increased our ability to predict secondary and tertiary folds from primary sequence information. However, determining how these static structures are coupled to function, particularly in proteins not amenable to biophysical and structural analyses, remains a significant challenge. The integral membrane VirA histidine kinase of Agrobacterium tumefaciens is an example of remarkable signaling complexity controlling the very first commitments to pathogenesis. We have been able to predict the phenol-sensing linker domain as a GAF fold (Gao and Lynn, 2007), a structure type known to bind cyclic nucleotides, heme, simple chromophores, and branched-chain amino acids (Martinez et al., 2005; Handa et al., 2008), and to
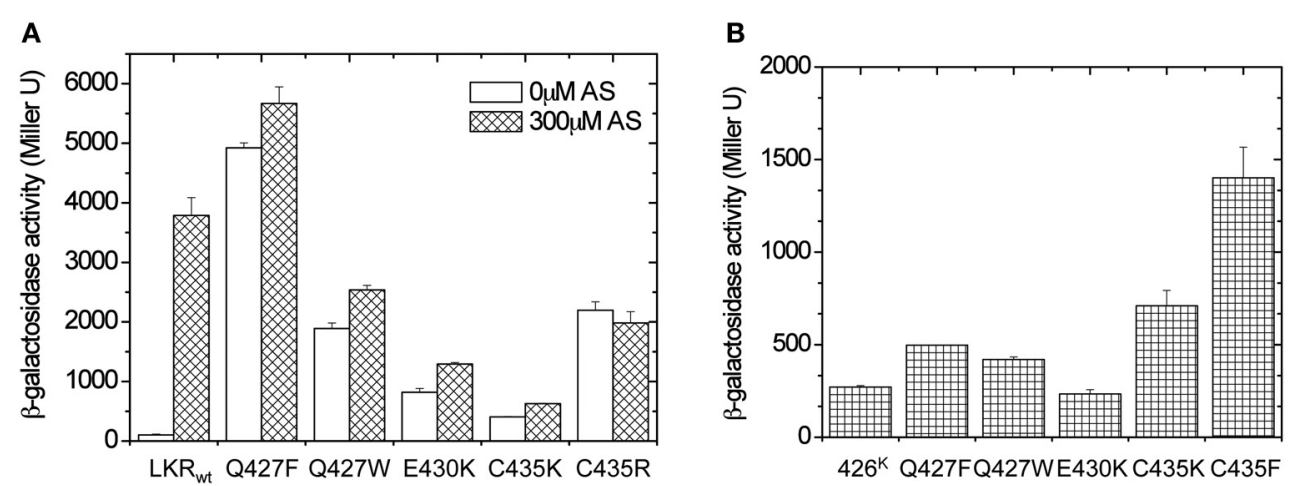

FIGURE 6 | Library screen for constitutive mutations within $\alpha \mathbf{4}$ (aa426-437). (A) $\beta$-galactosidase activity of the identified constitutively induced mutants. A. tumefaciens strain A136 carrying pRG109 and wild-type
LKR or LKR mutants were assayed for vir gene expression with or without $300 \mu \mathrm{M}$ AS. (B) A. tumefaciens strain A136 carrying pRG109 and wild-type $426^{\mathrm{K}}$ or $426^{\mathrm{K}}$ mutants were assayed for vir gene expression without inducers. 
A

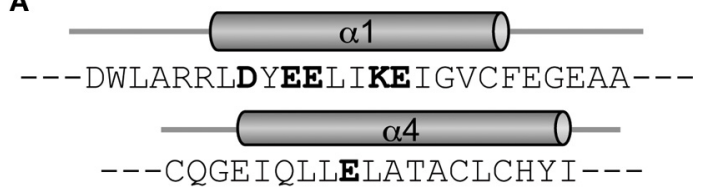

B

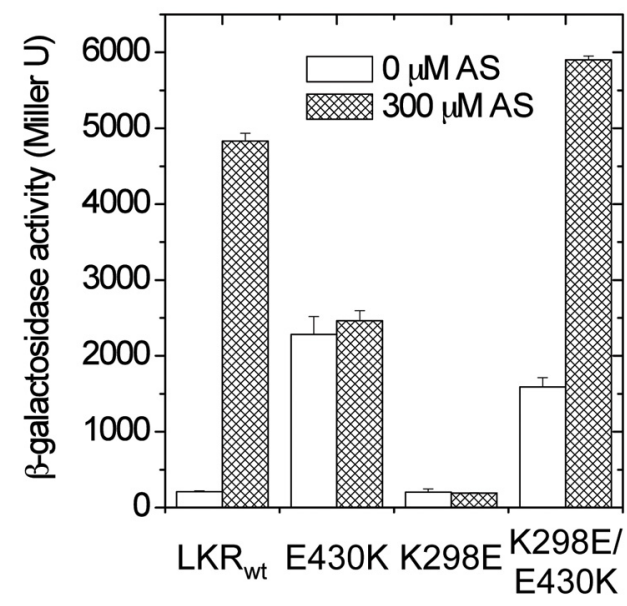

Expression:

FIGURE $7 \mid \alpha \mathbf{1}-\alpha \mathbf{4}$ salt-bridge formation. (A) The amino acids in linker $\alpha 1$ and $\alpha 4$. The charged residues are shown in bold. (B) A. tumefaciens strain A136 carrying pRG109 and LKR constructs with E430 and/or K298 mutants were assayed for $\beta$-galactosidase activity in the presence or absence of $300 \mu \mathrm{M}$ AS.

regulate secondary messenger metabolism (Sardiwal et al., 2005; Levdikov et al., 2006; Yang et al., 2008). The GAF domain is

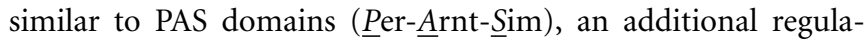
tory motif that is involved in protein functional control through interaction with a broad variety of small molecules (Ponting and Aravind, 1997; Hefti et al., 2004). Both GAF and PAS domains are observed in histidine kinases, with an estimated 9 and 33\% occurrence, respectively (Gao and Stock, 2009), and successful swaps of those signal sensing domains between different HK have been described (Kumita et al., 2003; Möglich et al., 2009), possibly indicating a common signaling mechanism. The developed structural model has now been used to examine the interactions regulating signal sensing and kinase activation of VirA. Specifically, the helix bundle architecture in the VirA linker and simple rotational motion mediated by these helices was proposed, and this mechanism has been further evaluated with a diverse series of fusions and chimeric constructs.

The GCN4 leucine zipper motif was used to anchor the orientation of the continuous helix proposed to connect the linker domain and the DHp domain of the kinase. When placed in the middle of the predicted coiled-coil region (aa450), "ON" and "OFF" conformations were identified that could be proposed to arise from different relative orientations of the helices (Figure 8A). Amino acid insertions at the coiled-coil suggested a clockwise rotation mediates VirA activation. The VirA histidine kinase employs a trans-phosphorylation mechanism
(Brencic et al., 2004), similar to the EnvZ histidine kinase in E. Coli (Cai and Inouye, 2003), meaning that the phosphorylation occurs across the subunits of the kinase dimer. A BLAST search identified VirA to have $24 \%$ identity to the Thermotoga maritima protein HK0853, whose entire cytoplasmic structure has been solved via X-ray crystallography (Marina et al., 2005). If VirA adopts a similar kinase fold as that of HK0853, the predicted clockwise rotation should bring the His474 in VirA closer to the ATP-binding domain of the other subunit for transphosphorylation (Figure 8A). This model is consistent with previous analyses (Gao and Lynn, 2007), suggesting the rotational motion controls kinase activity at the level of histidine phosphorylation rather than phosphoryl-transfer efficiency. However, HK0853 of T. maritima adopts a different cis-phosphorylation mechanism (Casino et al., 2009). The difference between VirA and HK0853 can be reconciled by the alignment of the coiled-coil region of both kinases (Figure 8B) and the proposed rotational mechanism. As shown in Figure 8B, the identified coiled-coil region of HK0853 is also located in front of the conserved $\mathrm{H}$ box (Marina et al., 2005). However, when compared with VirA, an additional residue in HK0853 exists between Gly466 and Thr467 of VirA. Having this extra residue in the coiled-coil would shift the conserved histidine of HK0853 (His260) from $e$ to $f$ in the heptads, which involves a movement of $+103^{\circ}$ relative to the position of His474 in VirA (Figure 8B). Therefore, the same rotational motion in HK0853 would move His 260 from an exposed surface to the ATP-binding domain of the same subunit, requiring a cis-phosphorylation mechanism (Figure 8B).

The observation of the high constitutive activities of $438^{\mathrm{K}}$ and $446^{\mathrm{K}}$ is consistent with the argument that the unimpeded kinase is constitutively active while regulatory domains successively repress this activity prior to signal perception release (McCullen and Binns, 2006). The kinase truncation results narrow the repressive region of the linker domain to aa426-437 (Figure 2C), and further lead to the hypothesis that the helical associations within the predicted helical bundle control the critical ON/OFF switch. An "OFF" interface is maintained in the un-induced state, and signal sensing switches it to the "ON" interface. Successful engineering of rotational motions at this region by similar GCN4 fusions displayed a clear rotational activation (Figure 5), and predicts the ON/OFF interface of $\alpha 4-\alpha 4^{\prime}$ (Figure 8A). Furthermore, the control by GCN4 at both $426^{\mathrm{K}}$ and $450^{\mathrm{K}}$ indicates the rotational motion is coherently transmitted from the linker domain to the kinase core. Indeed, library screens for constitutive mutants identified both hydrophobic and electrostatic interactions stabilizing the dimerization interface at $\alpha 4-\alpha 4^{\prime}$. A recent study on an engineered HK YF1 (generated by replacing the oxygen-sensing PAS domain of Bradyrhizobium japonicum FixL with the FMN (flavinmononucleotide)-binding LOV (light-oxygen-voltage) domain from Bacillus subtilis YtvA) provided structural insight into the coiled-coil motifs mediating signal transmission between functional domains (Diensthuber et al., 2013). Furthermore, it also implies a simple motion and a fundamental mechanism that can be shared between different signal sensing domains for kinase output.

And most interestingly, this search for functional long-range interactions identified the $\alpha 1$ helix as a key regulator for signal 
A
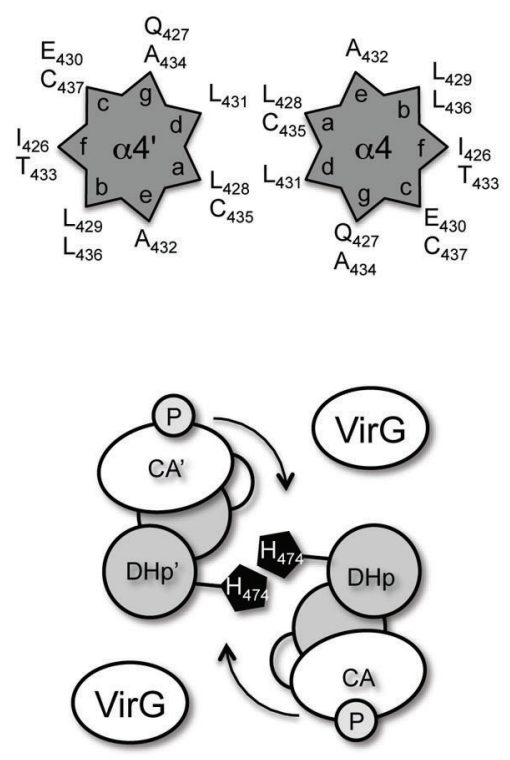

ON

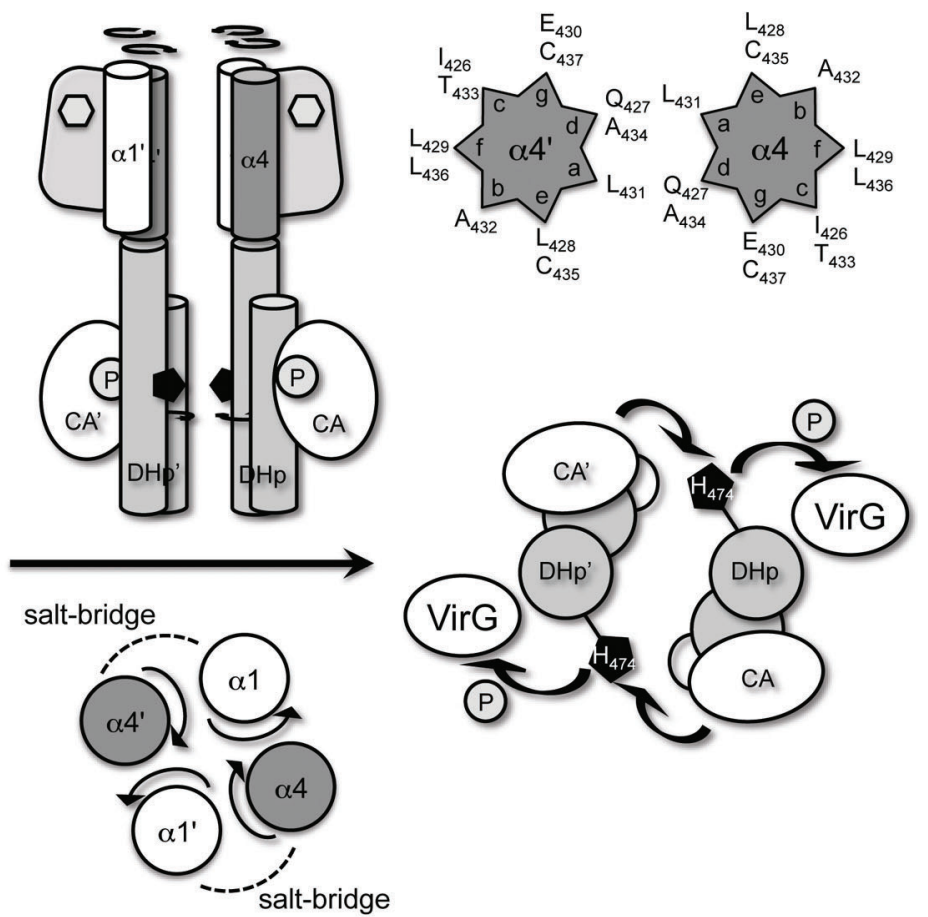

B

fgabcdefgabcdefg

$\operatorname{VirA}$

\section{TECDVLARRLEHAQRI
gabcdefgabcdef}

\section{TM0853 232 MENVTESKELERLKRIDRMKTEFIANISHELRTPLTAIK}
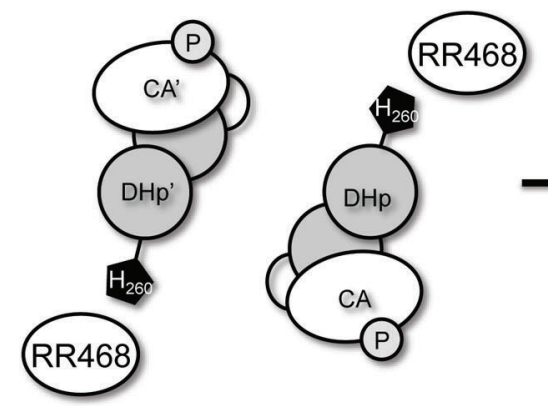

e

AHEFNNILGSIL

\section{$f$}

FIGURE 8 | VirA and Thermotoga maritima HK0853. (A) Proposed auto-phosphorylation mechanism of VirA, mediated by a 4 coiled-coil. The conserved His 474 of VirA, predicted to reside in the dimerization interface, is rotated clockwise upon phenolic sensing to close proximity of the ATP-binding domain at the other subunit for trans-phosphorylation and the subsequent VirG phosphoryl transfer. The ON and OFF $\alpha 4$ coiled-coil interface is represented in the helical wheel. (B) Sequence alignment of VirA and HK0853 at the coiled-coil region preceding the conserved histidine. The predicted heptads of the coiled-coil of both HK are shown from $a$ to $g$, and the conserved histidine are shown in bold. In TM0853, the additional residue in the kinase coiled-coil shifts the registry of the conserved His 260 by one residue, which creates a $+103^{\circ}$ displacement of His260 relative to VirA's His474. Therefore, the same proposed rotation upon signal sensing will move the conserved His260 in TM0853 toward the ATP-binding domain at the same subunit for cis-phosphorylation. activation in this rotational mechanism. Salt-bridge associations between $\mathrm{K} 298(\alpha 1)$ and $\mathrm{E} 430(\alpha 4)$ is consistent with the computational model of the helix bundle containing $\alpha 1$ and $\alpha 4$ interfaces in the VirA dimer (Wang et al., 2002) and its regulator role in signal transmission. The other charged residues in $\alpha 1$ were previously found to be important in controlling a "piston-like" motion, mediated by the monosaccharide/ $\mathrm{H}^{+}$sensing from the periplasmic domain (Gao and Lynn, 2007). Therefore, this bundle 
may be the conversion point for both sugar/pH and phenol inputs to counteract the repressive region in the dimerization interface at $\alpha 4-\alpha 4^{\prime}$. In addition, preliminary chemical cross-linking results aimed at clarifying the receiver domain's role in enhancing signal response precision indicated an association with the kinase core at this coiled-coil region (Figure S4), but the nature of this association is not yet clear.

The identified interactions point to highly cooperative longrange motions transmitting signal association within the VirA dimer to regulate the very first steps of pathogenesis. The positioning of $\alpha 1, \alpha 2$, and $\alpha 4$ vary in the three structural models for the GAF domain and indeed these kinds of structural details are the least well-defined in the structural algorithms. Figure 8 outlines a mechanistic model that is consistent with our chimeric fusion, but the nature of the long-range transmission (Gao and Stock, 2009) has also implicated symmetry switching models (Moore and Hendrickson, 2012). A recent structural analysis identified a critical proline residue in CpxA that contributes to helix bending in that kinase (Mechaly et al., 2014), but that residue is not conserved in VirA. The range of constructs prepared here provide opportunities to identify constructs amenable to direct structural analyses and further evaluation of these models. Most importantly, the remarkably coordinated action of VirA in processing three separate input signals likely contributed significantly to the success of this pathogen. These constructs now allow the system to be simplified sufficiently to define which signal is being processed and to map the signaling landscape of the host wound site for commitment to pathogenesis.

\section{ACKNOWLEDGMENTS}

We are grateful for support from the National Science Foundation through the award IOS \# 1423862 and to W. Seth Childers for insight and advice. We would also like to thank Tariq Shour for his technical work. B. Daniel Pierce is supported in part from a National Institute of Health K12 GM000680 Fellowship in Research and Science Teaching (FIRST) Institutional Research and Academic Career Development Award (IRACDA).

\section{SUPPLEMENTARY MATERIAL}

The Supplementary Material for this article can be found online at: http://www.frontiersin.org/journal/10.3389/fpls. 2014.00195/abstract

\section{REFERENCES}

Airola, M. V., Watts, K. J., Bilwes, A. M., and Crane, B. R. (2010). Structure of concatenated HAMP domains provides a mechanism for signal transduction. Structure 18, 436-448. doi: 10.1016/j.str.2010.01.013

Ankenbauer, R. G., and Nester, E. W. (1990). Sugar-mediated induction of Agrobacterium tumefaciens virulence genes: structural specificity and activities of monosaccharides. J. Bacteriol. 172, 6442-6446.

Aravind, L., and Ponting, C. P. (1999). The cytoplasmic helical linker domain of receptor histidine kinase and methyl-accepting proteins is common to many prokaryotic signalling proteins. FEMS Microbiol. Lett. 176, 111-116. doi: 10.1111/j.1574-6968.1999.tb13650.x

Brencic, A., Xia, Q., and Winans, S. C. (2004). VirA of Agrobacterium tumefaciens is an intradimer transphosphorylase and can actively block vir gene expression in the absence of phenolic signals. Mol. Microbiol. 52, 1349-1362. doi: 10.1111/j.1365-2958.2004.04057.x

Cai, S.-J., and Inouye, M. (2003). Spontaneous subunit exchange and biochemical evidence for trans-autophosphorylation in a dimer of Escherichia coli histidine kinase (EnvZ). J. Mol. Biol. 329, 495-503. doi: 10.1016/S0022-2836(03)00446-7
Cangelosi, G. A., Ankenbauer, R. G., and Nester, E. W. (1990). Sugars induce the Agrobacterium virulence genes through a periplasmic binding protein and a transmembrane signal protein. Proc. Natl. Acad. Sci. U.S.A. 87, 6708-6712. doi: 10.1073/pnas.87.17.6708

Casino, P., Rubio, V., and Marina, A. (2009). Structural insight into partner specificity and phosphoryl transfer in two-component signal transduction. Cell 139, 325-336. doi: 10.1016/j.cell.2009.08.032

Chang, C. H., and Winans, S. C. (1992). Functional roles assigned to the periplasmic, linker, and receiver domains of the Agrobacterium tumefaciens VirA protein. J. Bacteriol. 174, 7033-7039.

Chang, C. H., Zhu, J., and Winans, S. C. (1996). Pleiotropic phenotypes caused by genetic ablation of the receiver module of the Agrobacterium tumefaciens VirA protein. J. Bacteriol. 178, 4710-4716.

Cheung, J., and Hendrickson, W. A. (2010). Sensor domains of twocomponent regulatory systems. Curr. Opin. Microbiol. 13, 116-123. doi: 10.1016/j.mib.2010.01.016

Chillemi, G., Fiorani, P., Benedetti, P., and Desideri, A. (2003). Protein concerted motions in the DNA-human topoisomerase I complex. Nucleic Acids Res. 31, 1525-1535. doi: 10.1093/nar/gkg242

Diensthuber, R. P., Bommer, M., Gleichmann, T., and Möglich, A. (2013). Full-length structure of a sensor histidine kinase pinpoints coaxial coiled coils as signal transducers and modulators. Structure 21, 1127-1136. doi: 10.1016/j.str.2013.04.024

Duban, M. E., Lee, K., and Lynn, D. G. (1993). Strategies in pathogenesis: mechanistic specificity in the detection of generic signals. Mol. Microbiol. 7, 637-645. doi: 10.1111/j.1365-2958.1993.tb01155.x

Falke, J. J., and Hazelbauer, G. L. (2001). Transmembrane signaling in bacterial chemoreceptors. Trends Biochem. Sci. 26, 257-265. doi: 10.1016/S09680004(00)01770-9

Farago, B., Li, J., Cornilescu, G., Callaway, D. J. E., and Bu, Z. (2010). Activation of nanoscale allosteric protein domain motion revealed by neutron spin echo spectroscopy. Biophys. J. 99, 3473-3482. doi: 10.1016/j.bpj.2010.09.058

Gao, R., and Lynn, D. G. (2005). Environmental pH sensing: resolving the VirA/VirG two-component system inputs for Agrobacterium pathogenesis. J. Bacteriol. 187, 2182-2189. doi: 10.1128/JB.187.6.2182-2189.2005

Gao, R., and Lynn, D. G. (2007). Integration of rotation and piston motions in coiled-coil signal transduction. J. Bacteriol. 189, 6048-6056. doi: 10.1128/JB.00459-07

Gao, R., and Stock, A. M. (2009). Biological insights from structures of two-component proteins. Annu. Rev. Microbiol. 63, 133-154. doi: 10.1146/annurev.micro.091208.073214

Gelvin, S. B. (2000). Agrobacterium and plant genes involved in T-DNA transfer and integration. Annu. Rev. Plant Physiol. Plant Mol. Biol. 51, 223-256. doi: 10.1146/annurev.arplant.51.1.223

Gelvin, S. B. (2006). Agrobacterium virulence gene induction. Methods Mol. Biol. 343, 77-84. doi: 10.1385/1-59745-130-4:77

Gruez, A., Libiad, M., Boschi-Muller, S., and Branlant, G. (2010). Structural and biochemical characterization of free methionine-R-sulfoxide reductase from Neisseria meningitidis. J. Biol. Chem. 285, 25033-25043. doi: 10.1074/jbc.M110.134528

Handa, N., Mizohata, E., Kishishita, S., Toyama, M., Morita, S., UchikuboKamo, T., et al. (2008). Crystal structure of the GAF-B domain from human phosphodiesterase 10A complexed with its ligand, cAMP. J. Biol. Chem. 283, 19657-19664. doi: 10.1074/jbc.M800595200

Hefti, M. H., Françoijs, K.-J., de Vries, S. C., Dixon, R., and Vervoort, J. (2004). The PAS fold. A redefinition of the PAS domain based upon structural prediction. Eur. J. Biochem. 271, 1198-1208. doi: 10.1111/j.1432-1033.2004.04023.x

$\mathrm{Hu}$, X., Zhao, J., Degrado, W. F., and Binns, A. N. (2013). Agrobacterium tumefaciens recognizes its host environment using ChvE to bind diverse plant sugars as virulence signals. Proc. Natl. Acad. Sci. U.S.A. 110, 678-683. doi: $10.1073 /$ pnas. 1215033110

Hulko, M., Berndt, F., Gruber, M., Linder, J. U., Truffault, V., Schultz, A., et al. (2006). The HAMP domain structure implies helix rotation in transmembrane signaling. Cell 126, 929-940. doi: 10.1016/j.cell.2006.06.058

Kelley, L. A., and Sternberg, M. J. E. (2009). Protein structure prediction on the Web: a case study using the Phyre server. Nat. Protoc. 4, 363-371. doi: 10.1038/nprot.2009.2

Kumita, H., Yamada, S., Nakamura, H., and Shiro, Y. (2003). Chimeric sensory kinases containing $\mathrm{O} 2$ sensor domain of FixL and histidine kinase domain 
from thermophile. Biochim. Biophys. Acta 1646, 136-144. doi: 10.1016/S15709639(02)00555-1

Laskowski, R. A., Gerick, F., and Thornton, J. M. (2009). The structural basis of allosteric regulation in proteins. FEBS Lett. 583, 1692-1698. doi: 10.1016/j.febslet.2009.03.019

Lee, K., Dudley, M. W., Hess, K. M., Lynn, D. G., Joerger, R. D., and Binns, A. N. (1992). Mechanism of activation of Agrobacterium virulence genes: identification of phenol-binding proteins. Proc. Natl. Acad. Sci. U.S.A. 89, 8666-8670. doi: 10.1073/pnas.89.18.8666

Levdikov, V. M., Blagova, E., Joseph, P., Sonenshein, A. L., and Wilkinson, A. J. (2006). The structure of CodY, a GTP- and isoleucine-responsive regulator of stationary phase and virulence in gram-positive bacteria. J. Biol. Chem. 281, 11366-11373. doi: 10.1074/jbc.M513015200

Lin, Y.-H., Binns, A. N., and Lynn, D. G. (2008). "The initial steps in Agrobacterium tumefaciens pathogenesis: chemical biology of host recognition," in Agrobacterium: From Biology to Biotechnology, ed V. Citovsky (New York, NY: Springer), 221-241.

Lupas, A., Van Dyke, M., and Stock, J. (1991). Predicting coiled coils from protein sequences. Science 252, 1162-1164. doi: 10.1126/science.252.5009.1162

Marina, A., Waldburger, C. D., and Hendrickson, W. A. (2005). Structure of the entire cytoplasmic portion of a sensor histidine-kinase protein. EMBO J. 24, 4247-4259. doi: 10.1038/sj.emboj.7600886

Martinez, S. E., Bruder, S., Schultz, A., Zheng, N., Schultz, J. E., Beavo, J. A., et al. (2005). Crystal structure of the tandem GAF domains from a cyanobacterial adenylyl cyclase: modes of ligand binding and dimerization. Proc. Natl. Acad. Sci. U.S.A. 102, 3082-3087. doi: 10.1073/pnas.0409913102

McCullen, C. A., and Binns, A. N. (2006). Agrobacterium tumefaciens and plant cell interactions and activities required for interkingdom macromolecular transfer. Annu. Rev. Cell Dev. Biol. 22, 101-127. doi: 10.1146/annurev.cellbio.22.011105.102022

Mechaly, A. E., Sassoon, N., Betton, J.-M., and Alzari, P. M. (2014). Segmental helical motions and dynamical asymmetry modulate histidine kinase autophosphorylation. PLoS Biol. 12:e1001776. doi: 10.1371/journal.pbio.1001776

Melchers, L. S., Regensburg-Tuïnk, A. J., Schilperoort, R. A., and Hooykaas, P. J. (1989). Specificity of signal molecules in the activation of Agrobacterium virulence gene expression. Mol. Microbiol. 3, 969-977. doi: 10.1111/j.13652958.1989.tb00246.x

Miller, J. H. (1972). Experiments in Molecular Genetics. New York, NY: Cold Spring Harbor.

Mitrophanov, A. Y., and Groisman, E. A. (2008). Signal integration in bacterial two-component regulatory systems. Genes Dev. 22, 2601-2611. doi: 10.1101/gad.1700308

Möglich, A., Ayers, R. A., and Moffat, K. (2009). Design and signaling mechanism of light-regulated histidine kinases. J. Mol. Biol. 385, 1433-1444. doi: 10.1016/j.jmb.2008.12.017

Moore, J. O., and Hendrickson, W. A. (2012). An asymmetry-to-symmetry switch in signal transmission by the histidine kinase receptor for TMAO. Structure 20, 729-741. doi: 10.1016/j.str.2012.02.021

Pan, S. Q., Charles, T., Jin, S., Wu, Z. L., and Nester, E. W. (1993). Preformed dimeric state of the sensor protein VirA is involved in plantAgrobacterium signal transduction. Proc. Natl. Acad. Sci. U.S.A. 90, 9939-9943. doi: 10.1073/pnas.90.21.9939

Ponting, C. P., and Aravind, L. (1997). PAS: a multifunctional domain family comes to light. Curr. Biol. 7, R674-R677. doi: 10.1016/S0960-9822(06)00352-6

Sardiwal, S., Kendall, S. L., Movahedzadeh, F., Rison, S. C. G., Stoker, N. G., and Djordjevic, S. (2005). A GAF domain in the hypoxia/NO-inducible
Mycobacterium tuberculosis DosS protein binds haem. J. Mol. Biol. 353, 929-936. doi: 10.1016/j.jmb.2005.09.011

Stock, A. M., Robinson, V. L., and Goudreau, P. N. (2000). Twocomponent signal transduction. Annu. Rev. Biochem. 69, 183-215. doi: 10.1146/annurev.biochem.69.1.183

Tao, W., Malone, C. L., Ault, A. D., Deschenes, R. J., and Fassler, J. S. (2002). A cytoplasmic coiled-coil domain is required for histidine kinase activity of the yeast osmosensor, SLN1. Mol. Microbiol. 43, 459-473. doi: 10.1046/j.13652958.2002.02757.x

Tzfira, T., and Citovsky, V. (2006). Agrobacterium-mediated genetic transformation of plants: biology and biotechnology. Curr. Opin. Biotechnol. 17, 147-154. doi: 10.1016/j.copbio.2006.01.009

Wang, Y., Gao, R., and Lynn, D. G. (2002). Ratcheting up vir gene expression in Agrobacterium tumefaciens: coiled coils in histidine kinase signal transduction. Chembiochem 3, 311-317. doi: 10.1002/1439-7633(20020402)3:43.0.CO;2-N

Wang, Y., Mukhopadhyay, A., Howitz, V. R., Binns, A. N., and Lynn, D. G. (2000). Construction of an efficient expression system for Agrobacterium tumefaciens based on the coliphage T5 promoter. Gene 242, 105-114. doi: 10.1016/S03781119(99)00541-7

Watson, B., Currier, T. C., Gordon, M. P., Chilton, M. D., and Nester, E. W. (1975). Plasmid required for virulence of Agrobacterium tumefaciens. J. Bacteriol. 123, 255-264.

Winans, S. C., Kerstetter, R. A., and Nester, E. W. (1988). Transcriptional regulation of the virA and virG genes of Agrobacterium tumefaciens. J. Bacteriol. 170, 4047-4054.

Wise, A. A., Fang, F., Lin, Y. H., He, F., Lynn, D. G., and Binns, A. N. (2010). The receiver domain of hybrid histidine kinase VirA: an enhancing factor for vir gene expression in Agrobacterium tumefaciens. J. Bacteriol. 192, 1534-1542. doi: 10.1128/JB.01007-09

Wise, A. A., Voinov, L., and Binns, A. N. (2005). Intersubunit complementation of sugar signal transduction in VirA heterodimers and posttranslational regulation of VirA activity in Agrobacterium tumefaciens. J. Bacteriol. 187, 213-223. doi: 10.1128/JB.187.1.213-223.2005

Yang, X., Kuk, J., and Moffat, K. (2008). Crystal structure of Pseudomonas aeruginosa bacteriophytochrome: photoconversion and signal transduction. Proc. Natl. Acad. Sci. U.S.A. 105, 14715-14720. doi: 10.1073/pnas.0806718105

Zhang, J., Boone, L., Kocz, R., Zhang, C., Binns, A. N., and Lynn, D. G. (2000). At the maize/Agrobacterium interface: natural factors limiting host transformation. Chem. Biol. 7, 611-621. doi: 10.1016/S1074-5521(00)00007-7

Conflict of Interest Statement: The authors declare that the research was conducted in the absence of any commercial or financial relationships that could be construed as a potential conflict of interest.

Received: 31 January 2014; accepted: 23 April 2014; published online: 14 May 2014. Citation: Lin Y-H, Pierce BD, Fang F, Wise A, Binns AN and Lynn DG (2014) Role of the VirA histidine autokinase of Agrobacterium tumefaciens in the initial steps of pathogenesis. Front. Plant Sci. 5:195. doi: 10.3389/fpls.2014.00195

This article was submitted to Plant-Microbe Interaction, a section of the journal Frontiers in Plant Science.

Copyright (c) 2014 Lin, Pierce, Fang, Wise, Binns and Lynn. This is an open-access article distributed under the terms of the Creative Commons Attribution License (CC BY). The use, distribution or reproduction in other forums is permitted, provided the original author(s) or licensor are credited and that the original publication in this journal is cited, in accordance with accepted academic practice. No use, distribution or reproduction is permitted which does not comply with these terms. 